Department of

Gastroenterology and

Hepatology, University

Hospital Rotterdam,

the Netherlands

H L A Janssen

A Wijnhoud

F P Vleggaar

Department of

Gastroenterology and

Hepatology, University

Hospital Groningen,

the Netherlands

E B Haagsma

Department of Internal Medicine, University

Hospital St Radboud,

Nijmegen, the

Netherlands

S H M van Uum

Department of Gastroenterology, Free University Hospital,

Amsterdam, the

Netherlands

C M J van Nieuwkerk

Department of Gastroenterology,

University Hospital

Maastricht, the

Netherlands

R P Adang

Department of Gastroenterology, Academic Medical Centre, Amsterdam, the Netherlands

R A F M Chamuleau

Department of Gastroenterology, University Hospital

Utrecht, the

Netherlands

$\mathrm{J}$ van Hattum

Department of Clinical Epidemiology, Erasmus University Rotterdam, the Netherlands

B E Hansen

Department of Clinical Epidemiology, Leiden University Medical

Centre, the

Netherlands

F R Rosendaal

Department of Gastroenterology and Hepatology, Leiden

University Medical

Centre, the

Netherlands

B van Hoek

Correspondence to:

Dr H L A Janssen,

Department of

Gastroenterology and

Hepatology, Room Ca 326,

University Hospital

Rotterdam, Dr

Molewaterplein 40, 3015 GD

Rotterdam, the Netherlands.

devlaming@mdl.azr.nl

Accepted for publication 12 March 2001

\title{
Extrahepatic portal vein thrombosis: aetiology and determinants of survival
}

H L A Janssen, A Wijnhoud, E B Haagsma, S H M van Uum, C M J van Nieuwkerk, R P Adang, R A F M Chamuleau, J van Hattum, F P Vleggaar, B E Hansen, F R Rosendaal, B van Hoek

\begin{abstract}
Background-Malignancy, hypercoagulability, and conditions leading to decreased portal flow have been reported to contribute to the aetiology of extrahepatic portal vein thrombosis (EPVT). Mortality of patients with EPVT may be associated with these concurrent medical conditions or with manifestations of portal hypertension, such as variceal haemorrhage.
\end{abstract}

Patients and methods-To determine which variables have prognostic significance with respect to survival, we performed a retrospective study of 172 adult EPVT patients who were followed over the period 1984-1997 in eight university hospitals.

Results-Mean follow up was 3.9 years (range 0.1-13.1). Overall survival was $70 \%$ (95\% confidence interval (CI) $62-76 \%$ ) at one year, $61 \%(95 \% \mathrm{CI}, 52-67 \%)$ at five years, and $54 \%(95 \% \mathrm{CI}, 45-62 \%)$ at 10 years. The one, five, and 10 year survival rates in the absence of cancer, cirrhosis, and mesenteric vein thrombosis were $95 \%$ (95\% CI 87-98\%), 89\% (95\% CI 78-94\%), and $81 \%(95 \%$ CI $67-89 \%)$, respectively $(n=83)$. Variables at diagnosis associated with reduced survival according to multivariate analysis were advanced age, malignancy, cirrhosis, mesenteric vein thrombosis, absence of abdominal inflammation, and serum levels of aminotransferase and albumin. The presence of variceal haemorrhage and myeloproliferative disorders did not influence survival. Only four patients died due to variceal haemorrhage and one due to complications of a portosystemic shunt procedure.

Conclusion-We conclude that mortality among patients with EPVT is related primarily to concurrent disorders leading to EPVT and not to complications of portal hypertension.

(Gut 2001;49:720-724)

Keywords: extrahepatic portal vein thrombosis; portal vein obstruction; survival; mortality

The occurrence of extrahepatic portal vein thrombosis (EPVT) can be influenced by both local and systemic aetiological factors. Local factors comprise disorders leading to decreased portal flow such as liver cirrhosis and hepatobiliary malignancies. ${ }^{12}$ Systemic risk factors for EPVT consist mainly of acquired and inherited abnormalities leading to hypercoagulability. ${ }^{3}$ The clinical outcome of EPVT may be associated with these concomitant medical conditions or with manifestations of portal hypertension, in particular haemorrhage from ruptured oesophageal varices. Due to the rarity of the condition little is known about the determinants of survival and causes of death of patients with EPVT. ${ }^{4}$ The results of the few published studies on the prognosis of EPVT should be interpreted with caution as they either consist of a small number of patients or were performed in a highly selected population of patients from endoscopic or surgical units. ${ }^{56}$ To investigate variables associated with survival of EPVT, we undertook a retrospective analysis of a large cohort of adult patients, recruited from several medical disciplines.

\section{Patients and methods}

DESIGN OF THE STUDY

Patients were identified by means of a search in the computerised hospital registration system of all eight Dutch University Hospitals. All adult patients registered with a diagnosis of EPVT between January 1984 and July 1997 were included in the study. For all patients a standardised data form for specific clinical information, and confirmation of the diagnosis, was completed with data obtained from the medical charts by one of the authors (HJ). From a total of 236 patients thus identified with EPVT, 22 were excluded from analysis because the diagnosis was made at the postmortem examination. Forty two patients were excluded as they had been diagnosed prior to 1984 . Among the 172 patients in the cohort, follow up lasted from the time of diagnosis to either August 1997 or death, whichever came first. If outcome was unknown, family physicians were contacted. Five patients could not be traced and were considered lost to follow up (3\%). These patients were censored from the analysis at the last time point they were known to be alive. In four cases liver transplantation was carried out after the diagnosis of EPVT. For these patients the date of transplantation was considered as death due to liver failure. Separate analysis considering these four patients alive after transplantation revealed no alteration in the variables found to influence survival.

The following characteristics present at the time of diagnosis were evaluated to determine their prognostic significance for survival: age,

Abbreviations used in this paper: EPVT, extrahepatic portal vein thrombosis. 
Table 1 Risk factors of patients with extrahepatic portal vein thrombosis $(n=172)$

\begin{tabular}{|c|c|c|c|}
\hline Risk factor & $n$ & $\%$ & Specification \\
\hline Hepatic disorders & 52 & 30 & $\begin{array}{l}\text { Cirrhosis }(n=37) \text {, Budd-Chiari syndrome }(n=12) \text {, nodular regenerative hyperplasia } \\
(n=2) \text {, incomplete septal fibrosis }(n=1)\end{array}$ \\
\hline Abdominal inflammation & 30 & 17 & $\begin{array}{l}\text { Pancreatitis }(n=11) \text {, liver abscess }(n=7) \text {, inflammatory bowel disease }(n=6) \text {, cholangitis } \\
(n=3) \text {, umbilical vein infection }(n=1) \text {, appendicitis }(n=2)\end{array}$ \\
\hline Malignancies & 41 & 24 & $\begin{array}{l}\text { Hepatocellular carcinoma }(n=15) \text {, pancreatic carcinoma }(n=6) \text {, biliary tract carcinoma } \\
(n=3) \text {, other malignancy with liver metastases }(n=6) \text {, other malignancy without liver } \\
\text { metastases }(n=11)\end{array}$ \\
\hline Abdominal intervention & 40 & 23 & $\begin{array}{l}\text { Hepatobiliary surgery }(n=20) \text {, splenectomy }(n=13) \text {, liver transplantation }(n=3) \text {, jejunal } \\
\text { resection }(n=2) \text {, abdominal trauma }(n=2)\end{array}$ \\
\hline Hypercoagulability & 47 & 27 & $\begin{array}{l}\text { Pregnancy }(n=3) \text {, oral contraceptives }(n=13) \text {, paroxysmal nocturnal haemoglobinuria } \\
(n=4) \text {, antiphospholipid syndrome }(n=6) \text {, systemic lupus erythematosus }(n=2) \text {, factor } \\
\text { V Leiden mutation }{ }^{\star}(n=6) \text {, protrombin gene mutation }{ }^{\star}(n=3) \text {, protein } C \text { deficiency } \\
(n=7) \text {, protein } S \text { deficiency }(n=2) \text {, antithrombin deficiency }{ }^{\star}(n=1)\end{array}$ \\
\hline Myeloproliferative syndrome & 24 & 14 & $\begin{array}{l}\text { Polycythemia vera }(n=12) \text {, essential thrombocytosis }(n=3) \text {, myelofibrosis }(n=6) \text {, } \\
\text { myeloid leukaemia }(n=1) \text {, unclassified }(n=2)\end{array}$ \\
\hline
\end{tabular}

Patients can have more than one risk factor.

$\star$ Only tested for patients from whom a blood sample could be obtained in July $1997(n=73)$.

sex, oesophageal varices, variceal haemorrhage, ascites, hepatic encephalopathy, malignancy, liver cirrhosis, myeloproliferative disorders, mesenteric vein thrombosis, abdominal inflammation as a cause of EPVT, and levels of bilirubin, albumin, alanine aminotransferase, haemoglobin, and platelets.

DIAGNOSTIC ASSESSMENT

Diagnostic criteria for EPVT were partial or complete thrombotic obstruction of the extrahepatic portal vein, as documented by appropriate radiological abdominal imaging (that is, Doppler ultrasonography, computed tomography, magnetic resonance imaging, splenoportography), or laparotomy. The presence of oesophageal varices was evaluated by means of endoscopy, which had been performed in 130 cases $(76 \%)$. Patients with suspected variceal bleeding underwent endoscopic examination and, when indicated, sclerotherapy or band ligation. Ascites was diagnosed by physical examination and ultrasonography of the abdomen, which was carried out routinely. The presence of cirrhosis had to be proved by liver biopsy. A liver biopsy was obtained in 90 patients $(52 \%)$. In more than $95 \%$ of the nonbiopsied patients, no radiological signs of cirrhosis were found. Mesenteric vein thrombosis was defined as extensive thrombosis with clinical signs of intestinal infarction. Thus an asymptomatic thrombus limited to the confluent area of the superior mesenteric vein was not considered mesenteric vein thrombosis. A diagnosis of myeloproliferative disease was confirmed by bone marrow examination in all cases. Latent primary myeloproliferative disorder, as diagnosed by erythroid colony formation, was tested in a minority of patients and, for the purpose of this study, not considered to be a myeloproliferative disease. Standardised screening for thrombophilia was performed in a central laboratory for the 73 patients available for blood sampling. ${ }^{7}$ This screening included factor $\mathrm{V}$ Leiden mutation, prothrombin gene mutation, and inherited deficiencies for protein $\mathrm{C}$, protein $\mathrm{S}$, and antithrombin. Correction for potential acquired deficiencies of these coagulation inhibitors, as in liver failure, was done by correction for factor II and factor X. ${ }^{8}$ As only patients alive could be fully tested, thrombophilia was not included as a variable in the survival analysis.
STATISTICAL ANALYSIS

Survival was calculated by the Kaplan-Meier method. Duration of survival was determined irrespective of the cause of death. For univariate analysis, comparison of survival curves was based on the log rank test. Multivariate analysis was done by proportional hazard modelling. ${ }^{9}$ In order to avoid an excess of variables for the multivariate analysis, both cirrhotic and noncirrhotic patients diagnosed with Budd-Chiari syndrome were analysed as cirrhotics. The proportional hazard assumption of the Cox regression model was tested separately for each variable extending the multivariate model with a variable as a function of follow-up time- that is, a time dependent variable. Furthermore, interactions between explanatory variables were tested.

\section{Results}

Risk factors for the development of EPVT in the 172 investigated patients are shown in table 1. Systemic aetiological factors were present in $64(37 \%)$ and local aetiological factors in 110 (64\%) of the 172 patients. Coexistence of systemic and local risk factors was demonstrated in $28(16 \%)$ patients while more than one risk factor for EPVT, irrespective of whether they were systemic or local, was found in $54(31 \%)$ patients. In $27(16 \%)$ patients no risk factors for EPVT were identified.

Median age at diagnosis was 51 years (range 14-91). Fifty per cent of patients were male and more than $95 \%$ were Caucasian. An episode of bleeding from ruptured oesophagogastric varices was the initial manifestation in $52(30 \%)$ patients, $50(96 \%)$ of whom underwent endoscopic treatment. Mean follow up time for the 172 patients was 3.9 years (range 0.1-13.1). Sixty seven $(39 \%)$ patients died during follow

Table 2 Causes of death among 67 patients with extrahepatic portal vein thrombosis

\begin{tabular}{lrr}
\hline Cause of death & $n$ & $\%$ \\
\hline Hepatobiliary and gastrointestinal malignancies & 18 & 27 \\
Other malignancies & 12 & 18 \\
Liver failure & 10 & 15 \\
Cardiopulmonary disorders & 9 & 13 \\
Variceal bleeding & 4 & 6 \\
Infection & 4 & 6 \\
Postoperative complications & 2 & 3 \\
Other causes & 5 & 8 \\
Unknown & 3 & 4 \\
\hline
\end{tabular}




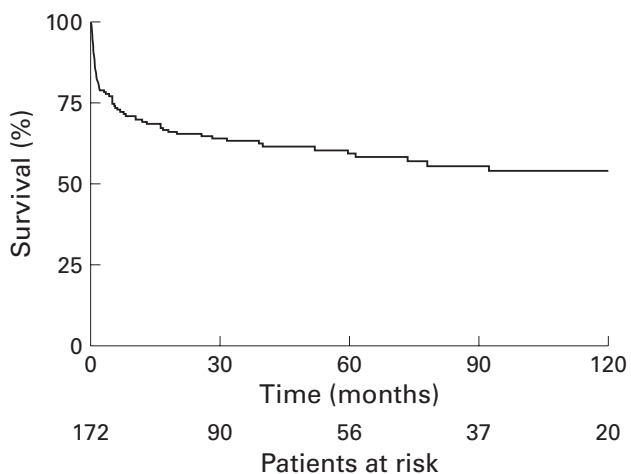

Figure 1 Actuarial survival of 172 patients with extrahepatic portal vein thrombosis. Number of patients at risk during follow up are shown along the $x$ axis.

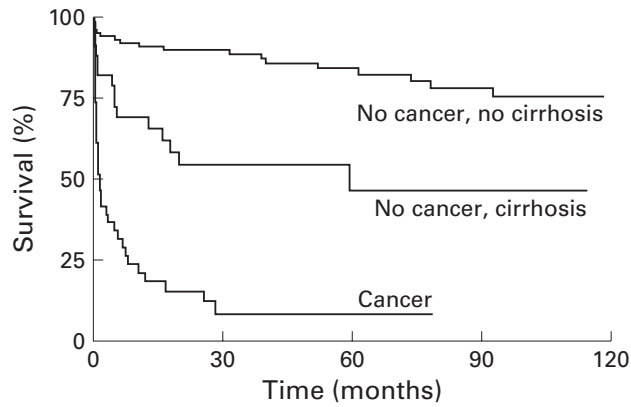

Figure 2 Actuarial survival rates for patients with extrahepatic vein thrombosis without cirrhosis and cancer (patients at risk $n=98, n=47$, and $n=17$ after 0, 5, and 10 years of follow up, respectively), for patients with cirrhosis but no cancer (patients at risk $n=33, n=9$, and $n=3$ after 0,5 and 10 years of follow up, respectively), and for patients with cancer (patients at risk $n=41$ and $n=3$ after 0 and 5 years of follow up, respectively).

up. The causes of death are listed in table 2 . Most patients died of (hepatobiliary) cancer and liver failure. Variceal bleeding was the cause of death in only four patients (2\%). Among the 98 patients with non-malignant and non-cirrhotic EPVT, two (2\%) died of variceal haemorrhage.

Overall survival was $70 \%$ (95\% confidence interval (CI) $62-76 \%)$ at one year, $61 \%(95 \%$ CI $52-67 \%)$ at five years, and $54 \%(95 \%$ CI $45-62 \%$ ) at 10 years (fig 1 ). The survival rates for EPVT patients without cancer and cirrhosis and for those with either cirrhosis or cancer is shown in fig 2 . The one, five, and 10 year survival rates in the absence of cancer, cirrhosis, and mesenteric vein thrombosis were $95 \%$ (95\% CI 87-98\%), 89\% (95\% CI 78-94\%), and $81 \%$ (95\% CI $67-89 \%)$, respectively $(n=83)$. From a total of 10 deaths in this group of 83 patients, only one died of variceal bleeding.

Patient characteristics at the time of diagnosis evaluated for their prognostic significance are shown in table 3. Variables significantly associated with reduced survival according to the univariate analysis were advanced age, cirrhosis, ascites, malignancy, absence of oesophageal varices, absence of abdominal inflammation, high levels of serum alanine aminotransferase, and low levels of serum albumin. Survival probability for patients with concurrent mesenteric vein thrombosis was markedly reduced but failed to reach significance. Neither the occurrence of variceal
Table 3 Characteristics at diagnosis of patients with extrahepatic portal vein thrombosis $(n=172)$ and results of univariate analysis

\begin{tabular}{|c|c|c|c|}
\hline & $n$ & $\begin{array}{l}5 \text { year } \\
\text { survival (\%) }\end{array}$ & $p$ Value ${ }^{\star *}$ \\
\hline \multicolumn{4}{|l|}{ Age (y) } \\
\hline$<40$ & 48 & 83 & \multirow[t]{3}{*}{$<0.001$} \\
\hline $40-60$ & 70 & 62 & \\
\hline$>60$ & 54 & 39 & \\
\hline \multicolumn{4}{|l|}{ Sex } \\
\hline Male & 86 & 58 & \multirow[t]{2}{*}{0.52} \\
\hline Female & 86 & 63 & \\
\hline \multicolumn{4}{|c|}{ Haemoglobin (mmol/l) } \\
\hline$<7.5$ & 76 & 58 & \multirow[t]{2}{*}{0.51} \\
\hline$\geqslant 7.5$ & 96 & 63 & \\
\hline \multicolumn{4}{|c|}{ Platelet count $\left(\times 10^{9} / 1\right)$} \\
\hline$<170$ & 84 & 56 & \multirow[t]{2}{*}{0.26} \\
\hline$\geqslant 170$ & 88 & 65 & \\
\hline \multicolumn{4}{|c|}{ Alanine aminotransferase (U/l) } \\
\hline$<30$ & 93 & 75 & \multirow[t]{2}{*}{$<0.001$} \\
\hline$\geqslant 30$ & 79 & 43 & \\
\hline \multicolumn{4}{|c|}{ Bilirubin $(\mu \mathrm{mol} / \mathrm{l})$} \\
\hline$<20$ & 86 & 66 & \multirow[t]{2}{*}{0.18} \\
\hline$\geqslant 20$ & 86 & 55 & \\
\hline \multicolumn{4}{|c|}{ Albumin (g/l) } \\
\hline$<35$ & 75 & 43 & \multirow[t]{2}{*}{$<0.001$} \\
\hline$\geqslant 35$ & 97 & 75 & \\
\hline \multicolumn{4}{|c|}{ Oesophageal varices ${ }^{\star}$} \\
\hline Absent & 26 & 49 & \multirow[t]{2}{*}{0.02} \\
\hline Present & 104 & 70 & \\
\hline \multicolumn{4}{|c|}{ Variceal bleeding } \\
\hline Absent & 120 & 67 & \multirow[t]{2}{*}{0.43} \\
\hline Present & 52 & 58 & \\
\hline \multicolumn{4}{|l|}{ Ascites } \\
\hline Absent & 107 & 68 & \multirow[t]{2}{*}{0.01} \\
\hline Present & 65 & 48 & \\
\hline \multicolumn{4}{|c|}{ Mesenteric vein thrombosis } \\
\hline Absent & 153 & 62 & \multirow[t]{2}{*}{0.08} \\
\hline Present & 19 & 41 & \\
\hline \multicolumn{4}{|l|}{ Cirrhosis } \\
\hline Absent & 124 & 68 & \multirow[t]{2}{*}{$<0.001$} \\
\hline Present & 48 & 35 & \\
\hline Myeloproli & diseas & & \\
\hline Absent & 148 & 56 & 0.12 \\
\hline Present & 24 & 88 & \\
\hline Malignancy & & & \\
\hline Absent & 131 & 77 & $<0.001$ \\
\hline Present & 41 & 8 & \\
\hline Abdominal & matior & & \\
\hline Absent & 143 & 55 & 0.001 \\
\hline Present & 29 & 92 & \\
\hline
\end{tabular}

$\star 130$ patients tested.

${ }^{\star}$ Log rank test.

bleeding nor the presence of myeloproliferative disorders at diagnosis influenced survival significantly. The results of multivariate analysis are presented for all investigated patients $(n=172)$ and for those with non-malignant EPVT $(n=131)$ in table 4. Age, absence of abdominal inflammation, cirrhosis, malignancy, mesenteric vein thrombosis, and serum levels of alanine aminotransferase and albumin had an independent prognostic value for survival. Test for the proportional risk assumptions did not show any non-proportionalities.

Forty six $(27 \%)$ of the 172 patients were treated with anticoagulation at their last hospital visit. Many were treated intermittently during follow up. Seventeen $(10 \%)$ of the 172 patients underwent a procedure to reduce portal hypertension. Fourteen had a portosystemic shunt (mesocaval $n=6$, splenorenal $n=5$, transjugular intrahepatic portosystemic shunt procedure $n=3$ ), one a thrombectomy, and two a portal reconstruction for EPVT as a consequence of liver transplantation. Median time between diagnosis of EPVT and intervention 
Table 4 Variables significantly influencing survival according to multivariate analysis. Relative risk (RR) of death is given for all investigated patients $(n=172)$ and for those with non-malignant extrahepatic portal vein thrombosis $(E P V T)(n=131)$

\begin{tabular}{|c|c|c|c|c|c|c|}
\hline & \multicolumn{3}{|c|}{$E P V T(n=172)$} & \multicolumn{3}{|c|}{$\begin{array}{l}\text { Non-malignant EPVT } \\
(n=131)\end{array}$} \\
\hline & $R R$ & $95 \% C I$ & $p$ Value & $R R$ & $95 \% C I$ & $p$ Value \\
\hline \multicolumn{7}{|l|}{ Age (y) } \\
\hline $40-60$ & 2.6 & $1.1-5.9$ & 0.02 & 1.4 & $0.5-3.8$ & 0.5 \\
\hline$>60$ & 5.8 & $2.5-13.2$ & $<0.001$ & 5.3 & $1.9-14.7$ & $<0.001$ \\
\hline Alanine aminotransferase $\geqslant 30 \mathrm{U} / 1$ & 1.9 & $1.1-3.2$ & 0.01 & 1.9 & $0.9-4.1$ & 0.06 \\
\hline Albumin $<35 \mathrm{~g} / 1$ & 3.2 & $1.8-5.8$ & 0.001 & 3.9 & $1.9-8.2$ & $<0.001$ \\
\hline Mesenteric vein thrombosis & 2.9 & $1.4-5.9$ & 0.003 & 7.4 & $2.7-20.1$ & $<0.001$ \\
\hline Cirrhosis & 3.9 & $1.9-8.1$ & 0.002 & 4.9 & $2.2-10.9$ & $<0.001$ \\
\hline Absence of abdominal inflammation & 4.6 & $1.3-15.7$ & 0.01 & 15.3 & $1.9-122.6$ & $<0.001$ \\
\hline Malignancy & 8.1 & $4.1-16.0$ & $<0.001$ & - & - & - \\
\hline
\end{tabular}

was one month (range 0-62). Five of the 17 patients died during follow up: one postoperatively, two from recurrent variceal haemorrhage, and two from causes unrelated to EPVT. For two of the deceased patients shunt occlusion was documented.

\section{Discussion}

In this study we identified causative and clinical factors that affected the survival of adult patients with EPVT. Previous studies on the prognosis of EPVT are scarce and are confined mainly to children or young adults with non-malignant and non-cirrhotic portal vein thrombosis. ${ }^{161011}$ Many of these studies were performed at a time when endoscopic therapy was not yet available. Moreover, these studies took place in single highly specialised hospital units which focused on the benefit of portosystemic shunting. However, patients with EPVT are encountered in several different medical disciplines and the present study was designed to minimise patient selection.

Over the last two decades it has become apparent that the aetiology of EPVT is highly diverse. We found at least one risk factor for the development of EPVT in $84 \%$ of patients. This despite the fact that not all patients underwent complete screening for hypercoagulability and that latent myeloproliferative disorders were not included as a risk factor. The occurrence of umbilical vein infection as a cause for EPVT was low as only newly diagnosed adult patients were investigated. Coexistence of causative factors was observed in about one third of patients, indicating that thrombosis of the portal vein, like other manifestations of thrombosis, can be a result of combined pathogenetic mechanisms. ${ }^{12}{ }^{13} \mathrm{~A}$ combination of systemic and local risk factors was found for $16 \%$ of the population. This suggests that in some patients genetic defects may create a predisposition for thrombosis which leads to clinically manifest EPVT in the presence of acquired thrombotic stimuli, such as cirrhosis, pancreatitis, splenectomy, and hepatobiliary surgery. ${ }^{74}$

An important finding of this study was the fact that mortality among patients with EPVT was related primarily to concurrent medical conditions, which are often the cause of EPVT, rather than to manifestations of portal hypertension, such as variceal haemorrhage. Prognosis was excellent if EPVT occurred in the absence of malignancy, cirrhosis, and mesenteric vein thrombosis. Absence of oesophageal varices was associated with a poor prognosis in the univariate analysis. This unexpected finding can be explained by the fact that many EPVT patients who suffered from life threatening diseases, such as cancer and mesenteric vein thrombosis, did not have the life expectancy to develop varices. The presence of variceal bleeding at presentation did not influence survival, and fatal haemorrhages occurred in only $2 \%$ of the investigated population. Even among patients with non-malignant and noncirrhotic EPVT only $1 \%$ experienced fatal variceal bleeding. On the one hand this finding contrasts with previous prognostic studies on EPVT in which variceal bleeding was held responsible for the death of $15-25 \%$ of patients. ${ }^{15}$ On the other hand it probably underlines the efficacy of endoscopic intervention which was employed as firstline treatment in $96 \%$ of our patients who presented with variceal haemorrhage. The success of endoscopic therapy for EPVT induced bleeding varices has already been documented. Groups from India and the Netherlands reported a negligible death probability due to recurrent EPVT induced variceal bleeding five years after sclerotherapy. ${ }^{15}{ }^{16}$ Merkel et al found that in comparison with patients with variceal bleeding due to cirrhosis, the outcome of bleeding for patients with non-cirrhotic EPVT was significantly better. ${ }^{17}$ This phenomenon can be explained by intact coagulation and liver function in patients with non-cirrhotic EPVT and possibly by the development of porto-portal collaterals which in time may stabilise portal pressure.

Only $17(10 \%)$ of our 172 patients underwent a procedure to reduce portal hypertension (portosystemic shunting, portal thrombectomy, or portal reconstruction). Although the low number of shunts is partly related to the high prevalence of comorbidity in our population, it may also indicate the efficacy of endoscopic treatment in the secondary prevention of variceal bleeding. As most of the shunted patients were highly selected, our data were insufficient to analyse whether portosystemic shunting could influence survival. Excellent long term survival rates without occurrence of post-shunt encephalopathy have been described for patients exclusively treated with surgical shunts in the period prior to the introduction of endoscopic treatment. ${ }^{11}{ }^{19}$ However, randomised controlled studies are lacking and the long survival found in some of these studies could also be explained by selection of patients without considerable comorbidity. We also did not have the possibility of evaluating whether anticoagulation is beneficial for patients with EPVT. ${ }^{20}$ About $25 \%$ of our patients were treated with anticoagulation. Most were treated intermittently during follow up and in some no anticoagulation was given at diagnosis. The prognostic effect of both anticoagulation and portosystemic shunting should ideally be investigated in controlled prospective studies. 
In summary, our data indicate that the outcome of EPVT is primarily associated with concomitant diseases leading to EPVT and not with bleeding from ruptured oesophageal varices. Our findings support sclerotherapy or band ligation as the primary treatment modality for variceal bleeding as a result of EPVT. Portosystemic shunting should probably be reserved for patients without important comorbidity who fail to respond to repeated endoscopic treatment.

We are indebted to SW Schalm and HR van Buuren (Department of Hepatogastroenterology, University Hospital Rotterdam), and to JP Vandenbroucke (Department of Clinical Epidemiology, Leiden University Medical Centre) for their helpful advice. This study was supported by a grant from the Gastroshunt Foundation.

\section{Appendix}

In addition to the authors, the following investigators cooperated in the study: CBHW Lamers, PJ Kingma (Leiden University Medical Centre); JR Meinardi, PLM Jansen (University Hospital, Groningen); B Bouman (Free University Hospital, Amsterdam).

1 Webb LJ, Sherlock S. The aetiology, presentation and natural history of extrahepatic portal venous obstruction. $Q \mathcal{F}$ Med 1979;192:627-39.

2 Cohen J, Edelman RR, Chopra S. Portal vein thrombosis. A review. Am f Med 1992;92:173-82.

Valla D, Casadevall N, Huisse MG, et al. Etiology of portal vein thrombosis in adults. A prospective evaluation of primary myeloproliferative disorders. Gastroenterology 1988;94:1063-9.

4 Valla D, Condat B. Portal vein thrombosis in adults: pathophysiology, pathogenesis and management. F Hepatol 2000; 32:865-71.

5 Cardin F, Graffeo M, McCormick PA, et al. Adult "idiopathic" extrahepatic venous thrombosis. Importance of putative "latent" myeloloproliferative disorders and comparison with cases with known etiology. Dig Dis Sci comparison with 6 Belli L, Romani F, Sansalone CV, et al. Portal thrombosis in
cirrhotics. A retrospective analysis. Ann Surg 1986;203: cirrhotics.

7 Janssen HLA, Meinardi JR, Vleggaar FP, et al. Factor V Leiden mutation, prothrombin gene mutation and deficiencies in coagulation inhibitors associated with Budd-Chiari syndrome and portal vein thrombosis. Results from a collaborative case-control study. Blood 2000;96:2364-8.

8 Pabinger I, Allaart CF, Hermans J, et al. Hereditary protein $C$ deficiency: laboratory values in transmitters and guidelines for the diagnostic procedure. Thromb Haemost 1992;68:470-4.

9 Cox DR. Regression models and life tables. $\mathcal{F} R$ Stat Soc 1972;34:187-220.

10 Fonkalsrud EW, Myers NA, Robinson MJ. Management of extrahepatic portal hypertension in children. Ann Surg 1974;180:487-93.

11 Warren WD, Henderson JM, Millikian WJ, et al. Management of variceal bleeding in patients with noncirrhotic porment of variceal bleeding in patients with noncirr

12 Vandenbroucke JP, Koster T, Briët E, et al. Increased risk of venous thrombosis in oral contraceptive users who are carriers of factor V Leiden mutation. Lancet 1994;344:1453-7.

13 Rosendaal FR. Venous thrombosis: a multicausal disease. Lancet 1999;353:1167-73.

14 Denninger $\mathrm{MH}$, Chait Y, Casadevall N, et al. Cause of portal or hepatic venous thrombosis in adults: the role of multiple concurrent factors. Hepatology 2000;31:587-91.

15 Chawla YK, Dilawari JB, Ramesh GN, et al. Sclerotherapy in extrahepatic portal venous obstruction. Gut 1990;31: 213-16.

16 Vleggaar FP, Buuren HR van, Schalm SW. Endoscopic sclerotherapy for bleeding oesophagogastric varices secondary to extrahepatic portal vein obstruction in an adult Caucasian population. Eur 7 Gastroenterol Hepatol 1998;10: $81-5$.

17 Merkel C, Bolognesi M, Bellon S, et al. Long-term follow-up study of adult patients with non-cirrhotic obstruction of the portal system: comparison with cirrhotic patients. F Hepatol 1992;15:299-303.

18 Grauer SE, Schwartz SI. Extrahepatic portal hypertension. A retrospective analysis. Ann Surg 1979;189:566-72.

19 Voorhees AB, Price JB. Extraheptic portal hypertension. A retrospective analysis of 127 cases and associated clinical implications. Arch Surg 1974;108:338-41.

20 Lagasse JP, Bahallah ML, Salem N, et al. Thrombose aigue du systeme porte. Traitement par alteplase ou heparine seule chez 10 malades. Gastroenterol Clin Biol 1997;21:91923.

21 Condat B, Pessione F, Erlinger S, et al. Portal vein thrombosis. Outcome in 94 adult patients. Hepatology 1997;26: 204A.

\section{9th United European Gastroenterology Week}

The UEGW abstract book (Gut 2001;49(suppl III)) has again been produced as a CD-ROM and can be found attached to the inside back cover of this issue. 